\title{
Molecular Cloning of a Neurospora crassa Carotenoid Biosynthetic Gene (Albino-3) Regulated by Blue Light and the Products of the White Collar Genes
}

\author{
MARY ANNE NELSON,${ }^{1}+$ GIORGIO MORELLI,${ }^{2}$ ALESSANDRA CARATTOLI,${ }^{1}$ NICOLETTA ROMANO,${ }^{1}$ AND \\ GIUSEPPE MACINO ${ }^{1 *}$ \\ Dipartimento Biopatologia Umana, Sezione di Biologia Cellulare, Universita di Roma "La Sapienza”, Policlinico \\ Umberto I, I-00161, ' and Unita' di Nutrizione Sperimentale, Istituto Nazionale della Nutrizione, \\ via Ardeatina 546, I-00179,2 Rome, Italy
}

Received 29 August 1988/Accepted 19 December 1988

\begin{abstract}
The albino-3 (al-3) gene of Neurospora crassa, which probably encodes the carotenoid biosynthetic enzyme geranylgeranyl pyrophosphate synthetase, was cloned. The $N$. crassa triple mutant al-3 qa-2 aro-9 was transformed to $q a-2^{+}$with mixtures of plasmids bearing $N$. crassa DNA inserts, and the transformants were screened for the $a l-3^{+}$phenotype. One $a l-3^{+} q a-2^{+}$transformant (AL3-1) was examined in detail and shown to contain intact vector sequences integrated into the $N$. crassa genome. The vector and some flanking sequences were recovered from AL3-1 after restriction, ligation, and selection of chloramphenicol-resistant transformants of Escherichia coli. The flanking sequences were subsequently used to detect the al-3-containing plasmid in the mixture of about 1,800 plasmids. Restriction fragment length polymorphism mapping was carried out to confirm the identity of the cloned fragment. The level of the al-3 mRNA was shown to be increased 15-fold in light-induced (compared with that in dark-grown) wild-type mycelia. The light-dependent increase in al-3 mRNA levels was not observed in presumed regulatory mutant (white collar) strains.
\end{abstract}

Carotenoids are ubiquitous membrane pigments that play a major role in photoprotection, act as photosynthetic accessory pigments, and serve as precursors of vitamin A. They may also function as antitumor agents in humans (13). All plants and some microorganisms, both photosynthetic and nonphotosynthetic, synthesize carotenoids de novo. Carotenoids give the characteristic orange color to the asexual spores (conidia) of the filamentous fungus Neurospora crassa. Since carotenoids are not required for the growth of $N$. crassa, this organism is well suited for the study of the carotenoid biosynthetic enzymes and their regulatory products.

$N$. crassa responds in various ways to illumination by blue light at different phases of its life cycle. During vegetative growth, blue light stimulates carotenoid biosynthesis (15) and causes phase shifting and photosuppression of the circadian rhythm of conidiation (11). During the sexual cycle, blue light induces the formation of protoperithecia (the female reproductive structures) (7) and stimulates the positive phototropism of the perithecial beaks (14). Strains carrying mutations in either of two white collar genes ( $w$ ' -1 and $w c-2$ ) have been shown to be blind to blue light, and they are defective in all of the photoresponses described above $(8$, $9,14-16)$. The white collar genes probably encode regulatory products; they could encode the blue light photoreceptor or proteins necessary for the transcriptional activation of the target genes, among other possibilities.

Carotenoid biosynthesis is constitutive in the asexual spores of $N$. crassa but regulated by blue light in the mycelium $(6,15,16)$. The photoinduction of carotenogenesis in the mycelium requires the de novo synthesis of at least

\footnotetext{
* Corresponding author.

+ Present address: Department of Physiological Chemistry, University of Wisconsin-Madison, 1300 University Avenue, Madison, WI 53706.
}

three enzymes, the products of the albino genes (16). The light induction of carotenogenesis can be separated into two steps, photoactivation and the subsequent dark reactions (so called because they proceed irrespective of the light conditions). The dark reactions probably involve the de novo synthesis of carotenogenic enzymes, as suggested by studies with the protein synthesis inhibitor cycloheximide (25). Experiments with actinomycin $D$ have shown that the light response is likely to cause the transcriptional activation of the affected genes (30).

The carotenoid biosynthetic enzyme geranylgeranyl pyrophosphate (GGPP) synthetase catalyzes the conversion of isopentenyl pyrophosphate to GGPP. GGPP synthetase activity has been detected in a soluble enzyme fraction from wild-type $N$. crassa. When the soluble fraction is obtained from an albino-3 (al-3) mutant, GGPP synthetase activity is very low, suggesting that the al-3 gene is the structural gene for GGPP synthetase (16). We have isolated the al-3 gene, examined its expression in dark-grown and light-induced mycelia, and shown that the steady-state level of al-3 mRNA increases after the light treatment. Strains harboring mutations in either of the white collar genes failed to show this response to blue light, demonstrating a role for the $w c$ gene products in the light regulation of carotenoid biosynthesis.

\section{MATERIALS AND METHODS}

Strains of $N$. crassa. The following $N$. crass $a$ wild-type and mutant strains ( $a l$, albino; $w c$, white collar; qa, quinate; aro, aromatic cluster gene) were obtained from the Fungal Genetics Stock Center (FGSC; University of Kansas, Kansas City, Kans.): Oak Ridge wild-type 74-OR23A (FGSC no. 987), al-3 (RP100; FGSC no. 2082), wc-1 (MK2; FGSC no. 4403), wc-2 (ER33; FGSC no. 4407), and qa-2 aro-9 (M246 Y325M6; FGSC no. 3958). Restriction fragment length polymorphism (RFLP) mapping experiments were carried out in the laboratory of Robert L. Metzenberg (University of 
Wisconsin, Madison, Wis.) by using blots prepared from the DNAs of FGSC strains 4411, 4416, and 4450 through 4487. The al-3 qa-2 aro-9 (RP100 M246 Y325M6) strain was constructed by standard methods (5). Homokaryotic derivatives of the $a l-3^{+} q a-2^{+}$transformants were obtained by multiple successive single colony isolations. The presumed homokaryotic derivatives failed to segregate either al-3 or qa-2 conidia.

Recombinant plasmids. The plasmid clone bank containing inserts of $N$. crassa DNA in the pRAL1 vector was generously provided by Alan M. Lambowitz (Ohio State University; 1). This bank was maintained and propagated on the Escherichia coli HB101 strain. The pRAL1 vector contains the $q a-2^{+}$gene that serves as the selectable marker in transformations of $N$. crassa and the chloramphenicol resistance gene for selection in $E$. coli.

The transforming sequences from the AL3-1 transformant were recovered by transformation of $E$. coli to chloramphenicol resistance. A 10- $\mu \mathrm{g}$ sample of AL3-1 DNA was digested with HindIII or SalI, diluted in ligation buffer to $1 \mu \mathrm{g} / \mathrm{ml}$ (to avoid intermolecular annealing), and ligated with $1 \mathrm{U}$ of $\mathrm{T} 4$ DNA ligase per $\mathrm{ml}$. After ligation overnight at $4^{\circ} \mathrm{C}$, the mixtures were concentrated to $200 \mu \mathrm{l}$ with 2-butanol and ethanol precipitated. A $1-\mu \mathrm{g}$ sample of ligated DNA was used to transform $5 \times 10^{10}$ competent cells.

Transformation. Preparation of al-3 spheroplasts and their transformation with the pRALl clone bank were as described by Schweizer et al. (27). In early experiments, transformants were plated directly in regeneration agar, while in later experiments transformants were first regenerated in liquid medium (18) and then spread onto selection plates. Transformation of $E$. coli was carried out as described by Maniatis et al. (20).

Media and culture conditions. For photoinduction experiments, conidia were inoculated at $2 \times 10^{5}$ cells per $\mathrm{ml}$ in 100 ml of Vogel minimal medium (31) plus $2 \%$ sucrose and shaken in the dark at $30^{\circ} \mathrm{C}$ for 16 to $18 \mathrm{~h}$. Mycelia were harvested under red safelight illumination by filtration onto filter paper disks, washed with $100 \mathrm{ml}$ of sterile water at room temperature, and placed into petri dishes. Vogel minimal medium $(1 \mathrm{ml})$ was added to each culture, and then selected cultures were illuminated by applying $2.5 \mu \mathrm{W} / \mathrm{cm}^{2}$ in the blue region (measured on the surface of the mycelial mat) for the indicated times with two Sylvania GRO-LUX F 18W-GRO lamps.

Preparation of DNA and RNA. Plasmid DNA was prepared according to the method of Ish-Horowicz and Burke (17). $N$. crassa DNA was prepared as described by Sherman et al. (29), except that Novozym 234 (Biolabs, Novo Allé, Bagsvaerd, Denmark) was used instead of Zymolyase (Kirin Brewery, Tokyo, Japan). Total RNA was extracted from mycelia by using guanidinium thiocyanate as a protein denaturant (4) and was further purified by centrifugation through $\mathrm{CsCl}$ cushions (12). Polyadenylated RNA was isolated by chromatography on oligo(dT)-cellulose (2).

Northern (RNA) and Southern hybridization blots. RNA was denatured, electrophoresed on agarose gels containing formaldehyde (19), and transferred onto Hybond $\mathrm{N}$ filters. The ${ }^{32} \mathrm{P}$-labeled probes were prepared by using the random oligomer-primer method (10). Filters were hybridized at $48^{\circ} \mathrm{C}$ in $5 \times \mathrm{SSPE}(0.9 \mathrm{M} \mathrm{NaCl}, 0.05 \mathrm{M}$ sodium phosphate [pH 7.7], $0.05 \mathrm{M} \mathrm{Na}_{2}$ EDTA), $5 \times$ Denhardt solution $(0.1 \%$ bovine serum albumin, 0.1\% Ficoll [Pharmacia Fine Chemicals, Piscataway, N.J.], 0.1\% polyvinyl pyrrolidone), $50 \%$ formamide, $10 \%$ dextran sulfate, and $0.1 \%$ sodium dodecyl sulfate. Filters were washed at $58^{\circ} \mathrm{C}$ in $0.1 \times \mathrm{SSPE}-0.1 \%$ sodium dodecyl sulfate. Southern hybridizations were performed as described by Maniatis et al. (20).

\section{RESULTS}

Screening for $a l-3^{+}$transformants. The al -3 mutant used in this study makes extremely light orange conidia, in contrast to the intensely orange conidia of wild-type $N$. crassa strains; all al-3 mutants show similar leaky phenotypes. Spheroplasts prepared from the al-3 qa-2 aro-9 strain were transformed to $q a-2^{+}$with 10 plasmid pools, each containing about 1,800 different plasmids (1). Transformant colonies were allowed to conidiate, and the conidia were screened for production of carotenoids. About 10,000 stable $q a-2^{+}$transformants obtained with each of the 10 plasmid pools were examined in this way. Primary transformants producing light orange conidia (clearly more pigmented than the conidia produced by the al-3 mutant) were observed when plasmid pools 7 and 9 were used for transformation; these stable transformants were present very infrequently (from about 1 in 5,000 to 10,000 transformants). Several other transformants (obtained with plasmid pools 5, 7, and 9) were apparently abortive for the $a l-3^{+}$(but not the $q a-2^{+}$) phenotype in that the conidia they produced appeared significantly more pigmented in the initial selection plates while this phenotype was lost upon transfer and subsequent growth.

When initial attempts to isolate the $a l-3^{+}$gene using the sib selection procedure described by Akins and Lambowitz failed (for unclear reasons), another strategy for the isolation of the $a l-3^{+}$gene was pursued. A stable al $-3^{+}$transformant (called AL3-1), obtained with DNA from plasmid pool 9, was selected for this purpose. Homokaryotic derivatives of this transformant produced conidia with apparently normal amounts of carotenoid pigments. We tried to isolate part or all of the original transforming DNA from the AL3-1 transformant.

Isolation of chloramphenicol-resistant plasmids from the AL3-1 transformant by selection in $E$. coli. Chromosomal DNA from the AL3-1 transformant was probed in Southern hybridization experiments with a bacterial portion of the pRAL1 vector. In blots of undigested transformant DNA, plasmid sequences that had integrated into the chromosomal DNA were detected, and no free plasmids were observed. Southern blot analysis of digested AL3-1 DNA showed that the vector sequence was probably integrated in a single copy and had not been rearranged during the integrative transformation event (data not shown). Since pRAL1 contains unique HindIII and SalI sites that lie outside of essential portions of the vector, we used these two restriction enzymes in an attempt to rescue, in $E$. coli, plasmids containing the vector and flanking, potentially transforming, sequences.

Chromosomal DNA from the AL3-1 transformant was digested with either HindIII or SalI, ligated (in dilute solutions to avoid intermolecular annealing), and used to transform $E$. coli to chloramphenicol resistance. A single 5.2kilobase-pair (kbp) plasmid called pNC6 was isolated eight times from the Sall-digested AL3-1 DNA, and a single 10.8-kbp plasmid called $\mathrm{pNC} 3$ was isolated four times from the HindIII-digested transformant DNA. No other chloramphenicol-resistant transformants of $E$. coli were obtained in this experiment. The pNC6 and pNC3 plasmids were analyzed by conventional techniques. Their structures are shown in Fig. 1, and their properties are discussed further below. Both plasmids were used to transform al-3 qa-2 aro-9 (as described above), but all $q a-2^{+}$transformants continued 


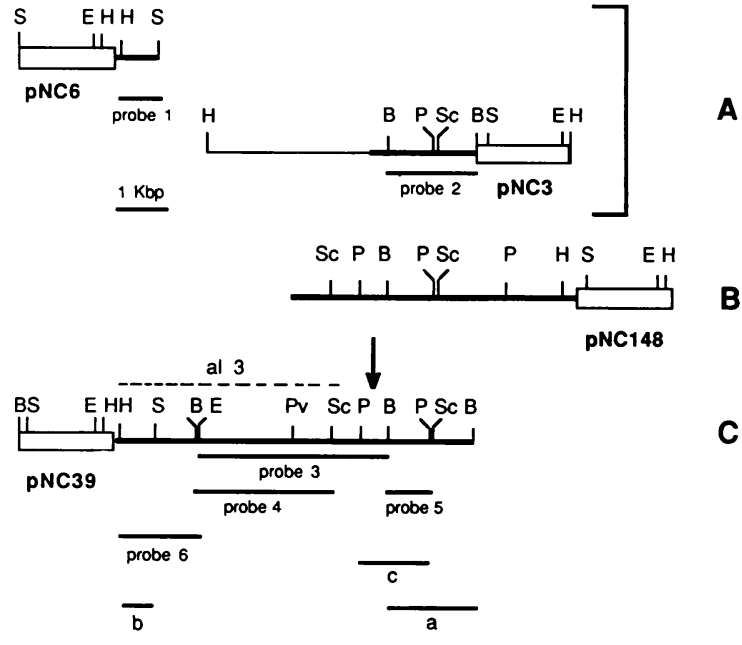

FIG. 1. Restriction maps of the al-3-containing and related plasmids. (A) Plasmids pNC6 and pNC3 which were isolated from transformant AL3-1 chromosomal DNA and selected by their ability to confer chloramphenicol resistance on $E$. coli transformants. (B) Plasmid pNC148, from plasmid pool 9, whose insert overlaps that of the al-3-containing pNC39. (C) Plasmid pNC39, the al-3-containing plasmid that was isolated from plasmid pool 9. Symbols: $\longrightarrow, N$. crassa sequences; $\square$, vector sequences;,$- N$. crassa genomic sequences that are present in pNC3 but not in the transforming plasmid pNC39. Abbreviations for restriction sites: P, PstI; Sc, SacI; E, EcoRI; B, BamHI; S, SalI; H, HindIII; Pv, PvuII. Numbered lines below sequences indicate probes that are discussed in the text, and lettered lines indicate restriction fragments. The $N$. crassa sequences are drawn to scale (indicated by the 1-kbp bar), but the vector sequences have been compressed. The AL3-1 transformant has two Bam HI fragments hybridizing with probe 5, a 5-kbp fragment that is also present in untransformed strains, and a $1.5-\mathrm{kbp}$ fragment (a) corresponding to that present in pNC3 and pNC39 (data not shown). The rightmost BamHI site of the $1.5-\mathrm{kbp}$ fragment was created during the construction of the $N$. crassa bank, when Sau3A partial digests of genomic DNA were cloned into the BamHI site of the vector (1); this BamHI site is not present in the wild-type strain, as is seen also upon examination of pNC148. The AL3-1 transformant contains the genomic 1.3-kbp PstI fragment (c) overlapping the 5-kbp BamHI fragment, as well as an additional $(4.5-\mathrm{kbp})$ fragment that was generated upon the insertion of pNC39 into the recipient genome (data not shown). The 3.4-kbp EcoRI-PstI fragment adjacent to the 1.3-kbp PstI fragment is intact in AL3-1. The arrow in Fig. 1C indicates the site at which pNC39 was integrated into the recipient genome. The approximate position of the al-3 gene is shown.

to manifest the al-3 phenotype, indicating that neither plasmid contains an intact, functional al-3 gene.

Isolation of a plasmid containing the entire al $-3^{+}$gene. DNA fragments from pNC3 and pNC6 were used to isolate the original transforming plasmid from pool 9 . When the approximately $700-\mathrm{kbp}$ HindIII-SalI restriction fragment from pNC6 (indicated as probe 1 in Fig. 1) was hybridized to blots of colonies from plasmid pool 9 , a single clone hybridizing to this probe was isolated 12 times; this clone was called pNC39. When the approximately $1.5-\mathrm{kbp}$ Bam HI fragment from pNC3 (Fig. 1, probe 2) was used to screen plasmid pool 9, pNC39 was again detected, as was a second plasmid, called pNC148.

The results of restriction mapping of the pNC3, pNC6, pNC39, and pNC148 plasmids are shown in Fig. 1. pNC6 is identical to the corresponding region of pNC39. pNC3 and pNC39 are colinear from the HindIII site within the vector to beyond the BamHI site within the $N$. crassa insert (and so both contain the 1.5-kbp BamHI restriction fragment a [Fig. 1]). pNC3 also contains a large (about $5 \mathrm{kbp}$ ) insert of $N$. crassa DNA from the site of the integrative transformation event. This analysis has confirmed that pNC39 was indeed the transforming plasmid that integrated into the AL3-1 genome and showed that no rearrangements of vector sequences occurred in the AL3-1 transformant. The related plasmid, pNC148, lacks the 1.5-kbp BamHI fragment a (Fig. 1) because the rightmost BamHI site (at the boundary between the vector and Neurospora DNA) was created by cloning (1).

To determine if one or both of these plasmids from pool 9 contained an intact $a l-3^{+}$gene, transformation experiments were carried out. pNC39 transformed al-3 qa-2 aro- 9 spheroplasts to $a l-3^{+} \mathrm{qa}^{-2^{+}}$at a high frequency (about $20 \%$ of the primary heterokaryotic transformants produced dark orange conidia, and the others produced conidia ranging in color from white to light orange). However, pNC148 was able to complement the $q a-2$ but not the al-3 mutation. Thus, by transformation we localized the putative al-3 gene to the pNC39 plasmid.

One of the problems that we encountered in the isolation of this nonselectable gene was the high number of clones (about 100,000 in the transformations with the 10 plasmid pools) that we had to screen. Reconstruction experiments have suggested that so many transformants had to be screened mostly due to the poor expression of the $a l-3^{+}$gene in the heterokaryotic primary transformants. Homokaryotic strains derived from most of the lightly pigmented primary transformants obtained with pNC39 produced dark orange conidia. However, several homokaryotic derivatives produced white or light orange conidia, suggesting the loss or the suboptimal expression of the $a l-3^{+}$gene.

RFLP mapping of the al-3 gene. The map position of the pNC39 insert in the $N$. crassa genome was determined by the RFLP technique that was devised by Metzenberg et al. (21; R. L. Metzenberg, and J. Grotelueschen, Neurospora Newsl. 34:39-44, 1987; R. L. Metzenberg, J. N. Stevens, E. U. Selker, and E. Morzycka-Wroblewska, Neurospora Newsl. 31:35-39, 1984). Briefly, this technique takes advantage of the large number of restriction site polymorphisms in two strains of $N$. crassa. By analyzing the segregation of conventional genetic markers and RFLPs, one can map any cloned fragment of $N$. crass $a$ DNA. An approximately 4-kbp EcoRI-BamHI fragment of pNC39 (Fig. 1, probe 3) was used as the probe for this analysis; the results are summarized in Table 1. The putative al-3 gene clearly maps on linkage group $\mathrm{V}$ between the cycloheximide-resistant $2($ cyh-2) and inositol ( $i n l$ ) genes, as expected on the basis of conventional genetic mapping with al-3 mutants (24). Single recombinants were detected between the $a l-3$ gene and these two flanking genes (Table 1, isolates A4 and I8, respectively), indicating a recombination frequency of about $3 \%$ between al-3 and each flanking marker. We consider it unlikely that we have isolated a closely linked suppressor of al-3 rather than the al-3 structural gene, although this remains a formal possibility.

Localization of the al-3 gene in pNC39. We had shown previously that the insert in pNC39 contains the intact $a l-3^{+}$ gene, while the overlapping insert in pNC148 does not. To more precisely localize the $a l-3$ gene, we transformed the al-3 qa-2 aro-9 strain with a modified pNC39 vector lacking the 1.3-kbp PstI fragment c (Fig. 1). Many of the primary $q a-2^{+}$transformants obtained with this modified vector formed light to dark orange pigmented conidia, indicating the presence of a functional $\mathrm{al}^{-3^{+}}$gene. These transformation 
TABLE 1. Segregation of genetic markers and RFLPs ${ }^{a}$

\begin{tabular}{|c|c|c|c|c|c|c|c|c|c|c|c|c|c|c|c|c|c|c|c|c|}
\hline \multirow{2}{*}{ Marker ${ }^{b}$} & \multicolumn{20}{|c|}{ Isolate no. } \\
\hline & A1 & A4 & B6 & B7 & $\mathrm{C} 1$ & $\mathrm{C} 4$ & D5 & D7 & E1 & E3 & E5 & E7 & $\mathrm{F} 1$ & F3 & G1 & G4 & H5 & H7 & 16 & 18 \\
\hline cyh-2 & $\mathbf{M}$ & 0 & $\mathrm{O}$ & 0 & $\mathbf{M}$ & $\mathrm{O}$ & $\mathrm{O}$ & $\mathbf{M}$ & $\mathrm{O}$ & $\mathrm{O}$ & $\mathbf{M}$ & $\mathbf{M}$ & $\mathbf{M}$ & $\mathrm{O}$ & $\mathrm{O}$ & $\mathrm{O}$ & $\mathbf{M}$ & $\mathbf{M}$ & $\mathrm{O}$ & $\mathrm{O}$ \\
\hline $\mathrm{pNC} 39^{c}$ & $\mathbf{M}$ & $\mathbf{M}$ & $\mathrm{O}$ & 0 & $\mathbf{M}$ & $\mathrm{O}$ & $\mathrm{O}$ & M & $\mathrm{O}$ & $\mathrm{O}$ & $\mathbf{M}$ & $\mathbf{M}$ & $\mathbf{M}$ & $\mathrm{O}$ & $\mathrm{O}$ & $\mathrm{O}$ & M & $\mathbf{M}$ & $\mathrm{O}$ & $\mathrm{O}$ \\
\hline inl & $\mathbf{M}$ & $\mathbf{M}$ & $\mathrm{O}$ & $\mathrm{O}$ & $\mathbf{M}$ & $\mathrm{O}$ & $\mathrm{O}$ & $\mathbf{M}$ & $\mathrm{O}$ & $\mathrm{O}$ & $\mathbf{M}$ & $\mathbf{M}$ & $\mathbf{M}$ & $\mathrm{O}$ & 0 & $\mathrm{O}$ & $\mathbf{M}$ & $\mathbf{M}$ & $\mathrm{O}$ & $\mathbf{M}$ \\
\hline
\end{tabular}

${ }^{a}$ Results are shown for 20 ordered progeny of the multicent-2- $a$ (in Oak Ridge genetic background) $\times$ Mauriceville-1c $A$ cross (abbreviated O and M, respectively). The three genes showed identical segregation in the other 18 progeny of this cross that were analyzed (data not shown).

${ }^{b}$ The three markers map on linkage group $\mathrm{V}$.

c Putative al-3 clone.

results have allowed the localization of the al-3 gene to a region of approximately $5 \mathrm{kbp}$ (Fig. 1).

Restriction analysis of AL3-1 genomic DNA localized the site of pNC39 integration into the host genome and confirmed that the al-3 region of pNC39 was intact in the AL3-1 transformant (Fig. 1). The integration site of pNC39 was localized between the PstI and BamHI sites (indicated by a heavy arrow in Fig. 1), as was suggested by analysis of pNC3, recovered from AL3-1 genomic DNA (described previously). Genetic mapping experiments showed that pNC39 was not integrated into the recipient genome adjacent or closely linked to the resident al-3 gene (data not shown).

Expression of the al-3 gene in light-induced and dark-grown mycelia. Since blue light induces the biosynthesis of carotenoids in $N$. crassa mycelia, and the al-3 gene probably encodes an early enzyme of this pathway (GGPP synthetase, whose activity has been shown to be induced by blue light [16]), we examined the expression of the al-3 gene in dark-grown and light-induced mycelia. A Northern (RNA) blot of RNA from wild-type mycelia (dark grown or exposed to continuous light for various times) showed that the approximately 2-kb al-3 mRNA is about $0-, 3-, 5-$, or 15 -fold more abundant in mycelia that have been exposed to light for $5,10,15$, or $30 \mathrm{~min}$, respectively (Fig. 2). The levels of the $\beta$-tubulin transcript, included here as a control, did not change significantly in response to light.

Blue light effects have been shown to be mediated by the products of the white collar genes $(8,9,14-16)$. To determine if the $w c$ gene products are necessary for the increased levels of al-3 mRNA in response to blue light, we examined the expression of the al-3 gene in dark-grown and light-induced

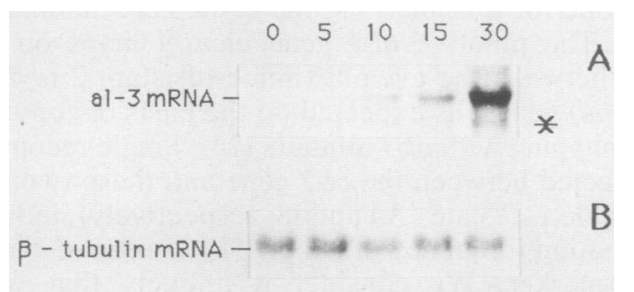

FIG. 2. Northern (RNA) blot showing the expression of the al-3 gene in dark-grown and light-induced mycelia. Total RNA was prepared from wild-type mycelia. Samples $(7.5 \mu \mathrm{g})$ of each RNA were separated on a $1.2 \%$ agarose- $6 \%$ formaldehyde gel, transferred to Hybond-N membrane, and hybridized to the al-3-specific probe (panel A). The position of the mitochondrial small ribosomal RNA (which showed some cross-hybridization with the al-3 probe) is indicated by an asterisk. The time of blue light induction is indicated as $0,5,10,15$, or $30 \mathrm{~min}$. Control hybridizations showed that the mRNA for the $\beta$-tubulin gene was present in similar amounts in each RNA preparation (panel B). The al-3 mRNA was detected with probe 4 (Fig. 1), and the $\beta$-tubulin mRNA was detected with pBT3 (22). mycelia of $w c-1$ and $w c-2$ mutant strains (Fig. 3). No difference in the abundance of the al-3 mRNA was detected after exposure of the $w c-1$ or $w c-2$ strain to light, indicating the inability of these strains to respond to blue light at this level. In these experiments, the putative al-3 mRNA was detected by using probe 4 (Fig. 1). Since probe 6 recognized the same mRNA species (data not shown) and no other transcripts were detected in these experiments, the approximately $2-\mathrm{kb}$ transcript probably corresponds to the $\mathrm{al}-3$ gene and not to an adjacent gene.

\section{DISCUSSION}

The cloning of the al-3 gene, which most likely encodes the carotenoid biosynthetic enzyme GGPP synthetase (16), has been described. Three lines of evidence suggest that the isolated gene indeed encodes the $a l-3$ protein. (i) The cloned sequence efficiently transforms the al-3 mutant strain to al $-3^{+}$. (ii) The RFLP mapping experiments showed that the cloned DNA maps to the expected location on linkage group V. (iii) The expression of the gene is regulated by blue light, as is the activity of the GGPP synthetase (16).

Heavy methylation of transforming and vector sequences at cytosine residues has been observed in $N$. crassa $(3,28)$. This methylation has been shown to be responsible for the failure to recover transforming sequences from $N$. crassa by selection in $E$. coli (23). The use of an $E$. coli strain that is deficient in the two methylcytosine restriction systems was sufficient to allow the direct recloning of methylated transforming sequences in $E$. coli (23). We were able to recover the $a l-3^{+}$transforming DNA without resorting to the use of

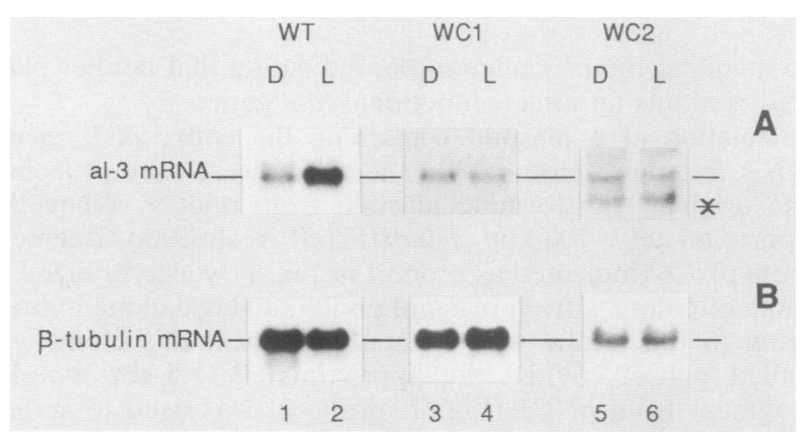

FIG. 3. Expression of the $a l-3$ gene in $w c-1$ and $w c-2$ mutants (WC1 and WC2, respectively). Wild-type (WT) and mutant strains were grown in the dark (D) or exposed to light for $15 \mathrm{~min}(\mathrm{~L})$. Lanes 1 to 4 contain $1 \mu \mathrm{g}$ of polyadenylated RNA; lanes 5 and 6 contain 10 $\mu \mathrm{g}$ of total RNA. Other experimental conditions were as described in Fig. 2 legend. The position of the mitochondrial small ribosomal RNA is indicated by an asterisk. 
restriction-deficient $E$. coli strains, suggesting that the methylation of transforming sequences might vary, depending perhaps on such variables as the transforming sequences themselves or the integration sites of these sequences into the genome (28).

Blue light induces the biosynthesis of carotenoids in $N$. crassa mycelia, and the activity of GGPP synthetase has been shown to increase after light treatment (16). We analyzed the expression of the $a l-3$ gene to determine the effect of light on the steady-state levels of the al-3 mRNA and found that the mRNA is about 15 -fold more abundant in mycelia exposed to light for $30 \mathrm{~min}$ than in dark-grown mycelia. This increase probably accounts for the increase in the enzymatic activity of GGPP synthetase after light treatment (16). Furthermore, the relatively high level of the al-3 transcript in dark-grown cultures is consistent with the enzymatic activity for the synthesis of GGPP found in dark-grown mycelia and with the accumulation of phytoene in the dark (16).

Biochemical studies have shown that the activities of GGPP synthetase, phytoene synthetase, and phytoene dehydrogenase are not induced by light in white collar mutants, leading to the hypothesis that $w c-1$ and $w c-2$ are regulatory mutants (16). We analyzed the effect of light on the expression of the al-3 gene in $w c-1$ and $w c-2$ mutant strains and did not observe any increase in the amount of al-3 mRNA after light treatment. Thus, our results have shown that the products of the white collar genes do indeed play a role in the regulation of al-3 gene expression, although the level at which this control is exerted remains to be determined.

New carotenoids are detectable about 30 min after irradiation (26). We found that a significant difference (threefold) in the level of the al-3 mRNA was established by 10 min after the beginning of the light treatment and that the accumulation of al-3 mRNA increased by 15 -fold after 30 min of light treatment. The experiments presented here clearly show that al-3 gene expression is regulated by light. Experiments with actinomycin D have suggested that the major control in the light induction of carotenogenesis is exerted at the level of transcription (30), suggesting that the light response entails an increased transcription of the al-3 gene. However, at this point it is not possible to exclude effects of light on al-3 mRNA stability. Mutagenesis of the al-3 promoter and the construction of chimeric genes using putative cis-regulatory regions derived from the al-3 gene could resolve these questions.

\section{ACKNOWLEDGMENTS}

We thank Alan M. Lambowitz for generously providing us with the Neurospora plasmid clone bank. We are grateful to Robert L. Metzenberg for assistance in the mapping of the cloned al-3 gene by RFLPs and for helpful discussions. We thank Tullio Aversa, Paola Ballario, and Elisa Di Nicola for their help and support, and Ida Ruberti for assistance in recloning the vector and al-3 transforming sequences.

This work was supported by grants from the Istituto PasteurFondazione Cenci Bolognetti and Progetti Finalizzati, Ingegneria Genetica del Consiglio Nazionale delle Ricerche.

\section{LITERATURE CITED}

1. Akins, R. A., and A. M. Lambowitz. 1985. General method for cloning Neurospora crassa nuclear genes by complementation of mutants. Mol. Cell. Biol. 5:2272-2278.

2. Aviv, H., and P. Leder. 1972. Purification of biologically active globin messenger RNA by chromatography on oligothymidylic acid-cellulose. Proc. Natl. Acad. Sci. USA 69:1408-1412.
3. Bull, J. H., and J. C. Wootton. 1984. Heavily methylated amplified DNA in transformants of Neurospora crassa. Nature (London) 310:701-704.

4. Chirgwin, J. M., A. E. Przybyla, R. J. MacDonald, and W. J. Rutter. 1979. Isolation of biologically active ribonucleic acid from sources enriched in ribonuclease. Biochemistry 18:5294 5299.

5. Davis, R. H., and F. J. de Serres. 1970. Genetic and microbiological research techniques for Neurospora crassa. Methods Enzymol. 17:79-143.

6. De Fabo, E. C., R. W. Harding, and W. Shropshire. 1976. Action spectrum between 260 and 800 nanometers for the photoinduction of carotenoid biosynthesis in Neurospora crassa. Plant Physiol. 57:440-445.

7. Degli Innocenti, F., and V. E. A. Russo. 1983. Photoinduction of protoperithecia in Neurospora crassa by blue light. Photochem. Photobiol. 37:49-51.

8. Degli Innocenti, F., and V. E. A. Russo. 1984. Isolation of new white collar mutants of Neurospora crassa and studies on their behavior in the blue light-induced formation of protoperithecia. J. Bacteriol. 159:757-761.

9. Degli Innocenti, F., and V. E. A. Russo. 1984. Genetic analysis of blue light-induced responses in Neurospora crassa, p. 213-219. In $\mathrm{H}$. Senger (ed.), Blue light effects in biological systems. Springer-Verlag KG, Berlin.

10. Feinberg, A. P., and B. Vogelstein. 1983. A technique for radiolabeling DNA restriction endonuclease fragments to high specific activity. Anal. Biochem. 132:6-13.

11. Feldman, J. F. 1982. Genetic approaches to circadian clocks. Annu. Rev. Plant Physiol. 33:583-608.

12. Glisin, V., R. Crkvenjakov, and C. Byus. 1974. Ribonucleic acid isolated by cesium chloride centrifugation. Biochemistry 13: 2633-2637.

13. Goodwin, T. W. 1986. Metabolism, nutrition, and function of carotenoids. Annu. Rev. Nutr. 6:273-297.

14. Harding, R. W., and S. Melles. 1983. Genetic analysis of phototropism of Neurospora crassa perithecial beaks using white collar and albino mutants. Plant Physiol. (Bethesda) 72:996-1000.

15. Harding, R. W., and W. Shropshire, Jr. 1980. Photocontrol of carotenoid biosynthesis. Annu. Rev. Plant Physiol. 31:217-238.

16. Harding, R. W., and R. V. Turner. 1981. Photoregulation of the carotenoid biosynthesis pathway in albino and white collar mutants of Neurospora crassa. Plant Physiol. (Bethesda) 68: 745-749.

17. Ish-Horowicz, D., and J. F. Burke. 1981. Rapid and efficient cosmid cloning. Nucleic Acids Res. 9:2989-2998.

18. Kuiper, M. T. R., R. A. Akins, M. Holtrop, H. de Vries, and A. M. Lambowitz. 1988. Isolation and analysis of the Neurospora crassa Cyt-21 gene. A nuclear gene encoding a mitochondrial ribosomal protein. J. Biol. Chem. 263:2840-2847.

19. Lehrach, H., D. Diamond, J. M. Wozney, and H. Boedtker. 1977. RNA molecular weight determinations by gel electrophoresis under denaturing conditions, a critical reexamination. Biochemistry 16:4743-4751.

20. Maniatis, T., E. F. Fritsch, and J. Sambrook. 1982. Molecular cloning: a laboratory manual. Cold Spring Harbor Laboratory, Cold Spring Harbor, New York.

21. Metzenberg, R. L., J. N. Stevens, E. U. Selker, and E. MorzyckaWroblewska. 1985. Identification and chromosomal distribution of 5S rRNA genes in Neurospora crassa. Proc. Natl. Acad. Sci. USA 82:2067-2071.

22. Orbach, M. J., E. B. Porro, and C. Yanofsky. 1986. Cloning and characterization of the gene for $\beta$-tubulin from a benomylresistant mutant of Neurospora crassa and its use as a dominant selectable marker. Mol. Cell. Biol. 6:2452-2461.

23. Orbach, M. J., W. P. Schneider, and C. Yanofsky. 1988. Cloning of methylated transforming DNA from Neurospora crassa in Escherichia coli. Mol. Cell. Biol. 8:2211-2213.

24. Perkins, D. D., A. Radford, D. Newmeyer, and M. Bjorkman. 1982. Chromosomal loci of Neurospora crassa. Microbiol. Rev. 46:426-570.

25. Rau, W. 1971. Light-dependent carotenoid synthesis. VII. Cy- 
cloheximide or anaerobic conditions as reversible blocks of the sequence of light-induced reactions. Planta (Berl.) 101:251-264.

26. Rau, W., and A. Rau-Hund. 1977. Light-dependent carotenoid synthesis. X. Lag-phase after a second illumination period in Fusarium aquaeductuum and Neurospora crassa. Planta (Berl.) 136:49-52.

27. Schweizer, M., M. E. Case, C. C. Dykstra, N. H. Giles, and S. R. Kushner. 1981. Identification and characterization of recombinant plasmids carrying the complete qa gene cluster from Neurospora crassa including the $q a-1^{+}$regulatory gene. Proc. Natl. Acad. Sci. USA 78:5086-5090.
28. Selker, E. U., B. C. Jensen, and G. A. Richardson. 1987. A portable signal causing faithful DNA methylation de novo in Neurospora crassa. Science 238:48-53.

29. Sherman, F., G. R. Fink, and C. W. Lawrence. 1978. Methods in yeast genetics, Cold Spring Harbor Laboratory, Cold Spring Harbor, New York.

30. Subden, R. E., and G. Bobowski. 1973. Evidence for actinomycin D inhibition of transcription of carotenoid loci in $\mathrm{Neu}$ rospora. Experientia 29:965-967.

31. Vogel, H. J. 1964. Distribution of lysine pathways among fungi: evolutionary implications. Am. Nat. 98:435-446. 